\title{
Cell Signaling During Trypanosoma cruzi Development in Triatominae
}

\author{
Mário A.C. Silva-Neto*, Patrícia Fampa, Carlo Donato Caiaffa, Alan B. Carneiro and \\ Georgia C. Atella
} Instituto de Bioquímica Médica at Universidade Federal do Rio de Janeiro, UFRJ, 21940-590, Rio de Janeiro, R.J.,
Brazil

\begin{abstract}
The year 2009 is the centennial anniversary of the original description of Chagas disease by Carlos Chagas. During the last 100 years, several advances have occurred regarding our knowledge of the development of Trypanosoma cruzi and its travel along the gut of Triatominae vectors. We have also witnessed the completion of both the human and parasite genome projects; the genome of one of Chagas disease vectors, Rhodnius prolixus, is currently being sequenced. The development of $T$. cruzi in triatomine gut relies on several biochemical and molecular processes. The biochemistry of blood digestion and the molecular and biological aspects of parasite development are well known. However, several signaling molecules are generated during blood digestion, and their effects on parasite biology are only beginning to be understood. Here, we will summarize our current knowledge in this area with an emphasis on heme and bioactive lipids. In addition, we will highlight some recently described members of the parasite signaling machinery, which were identified through high-throughput studies, but whose ligands are unknown thus far. Finally, we will discuss some potential aspects for future investigation in this area that may strengthen our view of such a concomitant biological process in the next years.
\end{abstract}

Keywords: Trypanosoma, heme, lysophosphatidylcholine, Chagas disease, Rhodnius.

\section{INTRODUCTION}

Chagas disease is caused by the trypanosomatid protozoan Trypanosoma cruzi. This disease was first described in the early twentieth century by Carlos Chagas, who reported its pathophysiological aspects and mechanism of transmission in 1909 [1-3]. Unfortunately, 100 years later, specific and efficient methods to block the transmission of this parasite and treat this condition still remain controversial [4]. The current number of patients infected with this disease is 11 million, with 200,000 new cases and at least 21,000 associated deaths each year [5].

The digestive system of Triatominae is generally composed of a pair of salivary glands, the anterior midgut (crop), the posterior midgut (small intestine) and the hindgut (rectum) [6]. After a meal, the ingested blood is stored in the crop and water is rapidly excreted by the Malpighian tubules [7]. Diuresis occurs early in the digestive process and concentrates the meal, which is slowly released from the anterior into the posterior midgut where enzymatic digestion occurs. The passage of the meal to the lumen of the posterior midgut is accompanied by the secretion of proteases, which are well characterized as amino peptidases, carboxypeptidases, cysteine proteases and aspartic proteases [8]. After a triatomine bug feeds on an infected mammalian host, the ingested trypomastigotes transform mostly into epimastigotes and some into spheromastigotes in the (triatomine) anterior midgut. In the posterior midgut, epimastigotes attach to perimicrovillar membranes (PMM), which are secreted by midgut epithelial

*Address correspondence to this author at the Instituto de Bioquímica Médica at Universidade Federal do Rio de Janeiro, UFRJ, 21940-590, Rio de Janeiro, R.J., Brazil; Tel: 5521 25626753; Fax: 5521 22708647;

E-mail: maneto@bioqmed.ufrj.br cells, and divide by binary division. This membrane system covers the midgut microvilli and extends towards its lumen [8].

Finally, once at the rectum, epimastigotes weakly attach to the hindgut cuticle and transform into metacyclic trypomastigotes, which are expelled with triatomine urine and feces and are then able to infect mammalian hosts [9-12].

The process of parasite differentiation relies on the attachment of parasites at different sites along the gut tissues of the vector as well as on the influence of soluble gut contents, whose constitution varies immensely along the course of the blood digestion process [12-13]. The attachment of $T$. cruzi epimastigotes to the PMM has been suggested to be important for parasite division [9]. Recently, the involvement of glycoinositolphospholipids (GIPLs), the major surface glycoconjugates of $T$. cruzi epimastigotes, in the adhesion of the parasite to the posterior midgut was demonstrated [14]. Despite the largely studied effects of GIPLs on vertebrate immune cells, the signaling pathways affected in the insect have not yet been studied. Adhesion of epimastigotes to the rectum surface is thought to be a prerequisite for differentiation into its infectious stages and involves hydrophobic interactions with the superficial cuticle layer of the hindgut [15-16]. It is not clear how adhesion triggers metacyclogenesis, but it must involve signaling pathways mediated by parasite surface receptors, which are activated by ligands present in vector tissues. In this respect, some glycoproteins present in the PMM seem to be parasite ligands, but their identities remain to be determined [17]. As blood digestion proceeds, both adhesion and metacyclogenesis per se are elicited by nutritional stress, which induces the expression of several stage-specific genes associated with the differentia- 
tion mechanism through a still unknown signaling pathway [18].

Establishment of a T. cruzi infection in an insect vector is dependent on and regulated by several physiological and biochemical factors (Fig. 1). Parasites enter the insect gut with the blood meal. In this context, they are submitted to drastic changes in temperature, $\mathrm{pH}$ and osmolarity and are exposed to saliva, gut contents and blood digestion products [12]. Some factors involved in the development of T. cruzi in the gut of the vector have been identified, including a lytic factor [19,20], lectins [21, 22] and hemoglobin constituents [23]. Other factors are suggested to trigger T. cruzi metacyclogenesis in vitro, including cAMP, cAMP analogues and adenylyl cyclase activators. Furthermore, intracellular levels of cAMP increase three- to four-fold before differentiation from epimastigotes to metacyclic trypomastigotes occurs [24]. In Rhodnius prolixus, cAMP excreted by Malpighian tubules and present in the urine might play a direct role in metacyclogenesis in vivo [25]. Vector hemolymph and intestine-derived components are able to promote metacyclogenesis as well. For example, an $\alpha$-D-globin-derived peptide isolated from Triatoma infestans is recognized by epimastigote surface receptors and stimulates parasite adenylyl cyclase, which leads to metacyclogenesis in vitro [26]. Subsequently, the effects of hemoglobin and synthetic peptides carrying $\alpha$-D-globin fragments on parasite growth and metacyclogenesis have also been demonstrated in vivo [27]. Therefore, the generation of biochemical mediators during blood digestion is clearly a central aspect of parasite development, as well as its growth and infection. In this review, we will emphasize signaling mediators derived from blood digestion that are involved in the regulation of the parasite life cycle in the vectpr. riatominae ingest five to ten times of their own weight in blood in a single meal [27, 28]. Hemoglobin digestion in the posterior midgut may induce an increase in heme concentration of up to $10 \mathrm{mM}$ (Fig. 1). In addition to forming reactive oxygen species, free heme can associate with phospholipid membranes to alter the structure of the lipid bilayer, leading to cell disruption $[29,30]$. Fenton's chemical reaction is a prime example of a harmful free radical reaction that is catalyzed by transition metals [31]. During Fenton's reaction, hydrogen peroxide, in the presence of iron (II), generates a highly reactive free radical species, the hydroxyl radical. Free heme creates a particular source of oxidative stress that may influence signaling pathways in surrounding cells. A close look at the antioxidant defense system in $R$. prolixus exposed the presence of superoxide dismutase (SOD), which catalyzes the dismutation of the superoxide radical $\left(\mathrm{O}_{2}{ }^{-}\right)$ [32], shows that this reaction produces hydrogen peroxide, which is, in turn, degraded by catalase (CAT). The highest SOD and CAT activities among all tested tissues were found in the posterior midgut. Injection of the CAT inhibitor 3amino-1,2,3-triazole (AT) promoted a dose-dependent inhibition of enzyme activity, with the concomitant production of high levels of hydrogen peroxide. Direct measurement of the hydrogen peroxide in this system was achieved in AT-treated insects. These results suggest that scavenging systems are important for antioxidant defense in the intestine. In this specific compartment of the vector digestive system, the processes of blood digestion and parasite differentiation are very advanced. It is tempting to speculate that the parasite must also display similar antioxidant defenses in order to face the redox environment of the intestine. However, such defenses have not yet been demonstrated.

Epimastigotes of $T$. cruzi actively multiply in the anterior midgut of the vector. Sequencing of the genome of this parasite revealed the absence of eight enzymes of the classical glycine-based heme synthesis pathway [33]. Heme-, hemoglobin- and globin-derived peptides were shown to induce parasite proliferation [34]. In the parasite, heme internalization probably occurs with an $\mathrm{ABC}$ transporter and in a region concentrated to the posterior end of the parasite that corresponds to reservosomes [34]. Reservosomes are phospholipid, ergosterol and cholesterol storage organelles and are localized to the posterior region of the epimastigote form of T. cruzi [35]. The content of these organelles is consumed during metacyclogenesis. It is likely that heme, as a lipophilic molecule, may induce some degree of lipid peroxidation as it is transported to the parasite and, once lipid mediators are generated, may influence parasite proliferation. While studies have shown that heme induced-signaling on parasite proliferation is solely mediated by calciumcalmodulin kinases (CaMKs), the role of a redox-mediated mechanism in this process has still not been assessed [36].

Studies in eukaryotes have determined two main families of proteins directly involved in signaling responses under redox stress: protein kinase $\mathrm{C}$ (PKC) and protein tyrosine phosphatase (PTP). PKC is a family of Ser/Thr protein kinases and is composed of at least 10 different isoforms. Its involvement with heme-induced oxidative stress was originally demonstrated in the regulation of the production of the anti-oxidant molecule urate by the fat body in $R$. prolixus [37]. Urate production is induced by cAMP and blocked by protein kinase A (PKA) inhibitors. Curiously, in the presence of heme, agonists of the cAMP pathway do not exert the same effects. In this situation, PKC is the enzyme responsible for the regulation of antioxidant production. However, the role of these pathways has not yet been tested in midgut epithelial cells, and the mechanisms, by which they regulate PKA- and PKC-mediated phosphorylation during blood digestion, if any, are not currently known.

The second family of signaling enzymes that may be involved in the regulation of cell signaling under redox conditions is the PTP family. These enzymes are involved in several intracellular signaling events, such as cell adhesion to substrate, cell metabolism and gene expression [38]. These enzymes are grouped into three different classes: protein tyrosine phosphatases (PTPs), Cdc25 and low molecular weight phosphatases (LMW-PTPs). They share a common motif (CX5R) in their catalytic site that allows for a cysteine-based catalytic cycle. In humans, the PTP group is subdivided into classical PTPs and dual specificity phosphatases (DSPs). Classical PTPs may be either receptortype or non-receptor-type. The unique catalytic reaction involving the SH group in the catalytic site implies possible modulation of PTPs by pro-oxidant molecules. Several redox states have been determined for these enzymes, which are strongly dependent on cell environment. Our group demonstrated that, with respect to blood-sucking arthropods, 
PTP regulates protein degradation during tick egg development [39]. Modulation of PTP gene expression occurs in some systems. Parasites traveling through the vector digestive system may be affected by redox-mediated signaling through both PKC and PTPs. A developmental control of surface ecto-phosphatase activity seems to occur in the epimastigote stage of $T$. cruzi, which displays the lowest enzyme activity in all tested stages [40]. Modulation of ecto-phosphatase activity at the $T$. rangeli surface by hydrogen peroxide has already been demonstrated [41]. Furthermore, it was demonstrated that Fenton's reaction affects the activity of another signaling molecule from the parasite's surface, an ecto-nucleoside triphosphate diphosphohydrolase in $T$. brucei brucei, the causative agent of African trypanosomiasis [42]. These studies revealed that the redox environment may finely tune the intracellular environment through the modification of signaling enzymes both in the vector and at the parasite surface. However, similar studies regarding vector and parasite PTPs, and surface enzymes of $T$. cruzi have not yet been conducted.

Typically, the average lipid concentration in vertebrate blood serum is $800 \mathrm{mg} / \mathrm{dL}$ [43]. Therefore, in a single blood meal a fifth instar bug may ingest close to $1 \mathrm{mg}$ of lipids which is equivalent of a $70 \mathrm{Kg}$ human to ingest close to $1 \mathrm{Kg}$ of lipid daily! Thus, in a typical blood meal, an adult bug or a fifth instar nymph may ingest approximately $2 \mathrm{mg}$ of lipids. The posterior midgut of the blood sucking triatomines is a major site of dietary lipid absorption. The digestion of neutral lipids, phospholipids and glycolipids generates a proportionally larger amount of free fatty acids that, upon absortion by midgut epithelial cells, will be used for de novo synthesis of diacylglycerols, triacylglycerols and phospholipids. The midgut provides lipids to be used in insect tissue development. The function of a hemolymphatic lipoprotein, lipophorin, distributes lipids to different organs, including the ovaries in order to attain their lipid demand during egg formation [44-46]. Usually, the complete digestion of a blood meal occurs over 12 days in Triatominae. Ten days after a blood meal, the digestion is almost complete, and there is a shift in the lipid metabolism in the posterior midgut. Injections of radiolabelled oleic acid (OA) have shown that the posterior midgut is the main organ that incorporates this lipid [46]. Furthermore, OA is incorporated into phospholipids and neutral lipids. These data indicate that, in the earlier phase of blood digestion and absorption, the insect posterior midgut provides lipids to the surrounding organs, but as digestion slows, the lipid flux is reversed and the posterior midgut receives lipids from the hemolymph [46]. It is possible that some of these lipids are incorporated into the PMM during their synthesis, which occurs at the initial phase of digestion (Fig. 1).

Trypanosomatids have incomplete de novo lipid synthesis and usually absorb lipids from the vertebrate bloodstream in order to meet their growth and differentiation requirements. Therefore, these pathogens critically rely on the presence of vertebrate lipoproteins, especially vertebrate LDL, to achieve infection. Despite this general and classical view of lipid metabolism in protozoan parasites, it is important to note that some differences may occur in the pathway with regard to cholesterol. Promastigotes of Leishmania synthesize a large amount of their sterols from leucine. T. brucei acquires phospholipids and sterols through endocytosis and intracellular processing of host LDL [47]. We have previously shown that $T$. rangeli, a trypanosomatid that reaches the vector hemolymph, uptakes the major hemolymphatic lipoprotein, lipophorin (Lp), from $R$. prolixus [48]. Also, we have shown lipid uptake by the malaria parasite during its life cycle in the invertebrate host [49]. Molecular evidence for the role of $\mathrm{Lp}$ in malaria parasite development has been provided [50]. However, the direct uptake of Lp and lipids by malaria parasite stages while inside its vector are not available. The published data provides evidence for the interaction of protozoan parasites with the lipid sources available in different insect environments. In this respect, the digestive system of triatomine bugs is a rich source of these molecules. Considering that these insects usually feed on huge amounts of blood, a massive amount of lipids is simultaneously ingested with trypanosomatids. A direct demonstration of such a pathway is still lacking for $T$. cruziinfected vector.

A PKC from T. cruzi epimastigotes was characterized, and its biochemical and immunological characteristics have been analyzed [51]. The involvement of this enzyme in sensing the lipid environment and its role in the parasite was revealed upon the demonstration that treatment of epimastigotes with intestinal homogenate from Triatoma infestans led to an increase in intracellular calcium followed by parasite differentiation to the trypomastigote stage [52]. Protease treatment of the intestinal fraction did not abolish the induction of differentiation. This effect is restricted to the lipid fraction of intestine, especially to OA. This fatty acid stimulates the de novo synthesis of diacylglycerol and the activation of PKC, which leads to parasite differentiation [53]. OA is able to trigger intracellular calcium in epimastigotes, inducing the translocation of several PKC isoforms, such as $\alpha$, $\beta, \gamma$ and $\delta$. PKC inhibitors abrogated both parasite differentiation and the membrane translocation of various PKC isoforms [54]. It will be interesting, in the future, to test the effects of heme-mediated lipid peroxidation in this OA fraction, specifically for their ability to promote $\mathrm{PKC}$ regulation in parasite differentiation (Fig. 1).

Some studies have concluded that the production of hydroxyl radicals can occur from free heme either in solution or bound to hemoglobin [55]. However, there is no evidence indicating that lipid peroxidation induced by heme occurs during blood digestion. Furthermore, lipid hydroperoxides may be formed during lipid peroxidation, which is initiated by another source of reactive species, such as $\mathrm{O}_{2}^{-}$formed either by NADPH oxidase or during mitochondrial respiration. This fact suggests that the pro-oxidant action of heme can be best described as an amplification of previously formed reactive species and depends on the occurrence and magnitude of other sources of free radicals [56]. In conclusion, despite the presence of the antioxidant enzyme system described above, lipid peroxidation may occur as a result of high levels of free heme and production of radical species from other sources. It is strikingly possible that lipid peroxidation products could be observed, for example, upon the inhibition of heme detoxification systems, such as hemeoxigenase. This interesting point should be addressed in the future. 


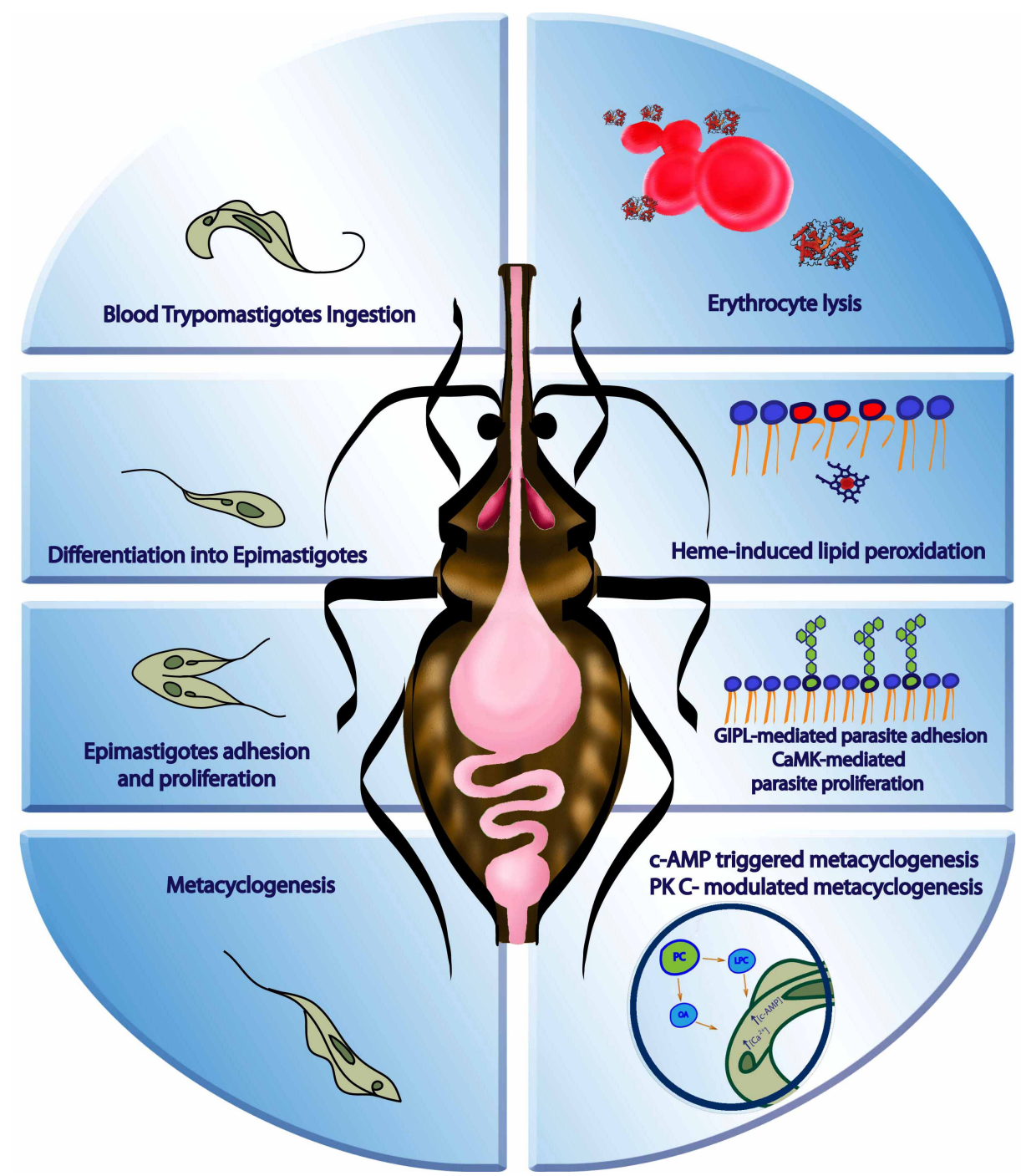

Fig. (1). Overview of major signaling events during $T$. cruzi development in Triatominae digestory system. Left panels, parasite development: Right panels, biochemical and signaling mechanisms. After a blood meal on an infected host, trypomastigotes transform mostly into epimastigotes and some into spheromastigotes in the anterior midgut, where following erythrocyte lysis, hemoglobin is gradually relased. Catalytic amounts of heme may attack unsaturated phospholipids from plasma lipoproteins producing several unknown lipid peroxidation products. In the posterior midgut, epimastigotes attach to perimicrovillar membranes through surface glycophosphatidylinositols and divide by binary division. Free heme modulates parasite proliferation through the activation of CaMK kinase. Finally, once at the hindgut, epimastigotes weakly attach to the rectal cuticle and transform into metacyclic trypomastigotes. Phospholipid-derived bioactive lipids such as oleic acid (OA) and eventually lysophosphophatidylcholine (LPC) regulate metacyclogenesis through generation of intracellular signaling molecules such as calcium and cyclic-AMP. Finally, parasites are expelled with triatomine urine and feces and are then able to infect mammalian hosts. The events in this cartoon have been already described in the literature and some of them are still under investigation. Please see the text for further details.

Usually phospholipids, mainly phosphatidylcholine, compose $40 \%$ of the ingested lipids in a blood meal. Blood feeding followed by the initiation of digestion may lead to the destruction of the blood cells and a release of large amounts of phospholipases, such as phospholipase $\mathrm{A}_{2}$ $\left(\mathrm{PLA}_{2}\right) . \mathrm{PLA}_{2}$ catalyzes the hydrolysis of the $s n-2$ fatty acyl bond of phospholipids, with the release of a free fatty acid and a lysophospholipid. Both of these products are important signaling molecules. We have shown the presence of lysophospholipids, mainly lysophosphatidylcholine (LPC), in the saliva and feces of $R$. prolixus [57,58]. LPC acts as a chemotactic molecule for monocytes and macrophages, but also plays a role in the production of NO by these cells, therefore enhancing $T$. cruzi infection through immunosuppression. Curiously, the phospholipase A1 was identified in the infective stages of $T$. cruzi, but not in epimastigotes [59]. This finding suggests that the parasite may rely on LPC that is derived from the insect, from the host and from its own enzymatic system. However, future tests on the ability of lysophospholipids derived from the parasite itself to immunosupress host cells as compared to that generated by $\mathrm{PLA}_{2}$ are required. Fatty acids released upon $\mathrm{PLA}_{2}$ hydrolysis, or present in the host blood and associated to albumin, may be processed by the eicosanoid pathway. Furthermore, proteomic analysis of epimastigote parasite forms identified the presence of prostaglandin F $2 \alpha$ 
synthase [60]. Prostaglandin $\mathrm{PGH}_{2}$ can be converted to the eicosanoid thromboxane $\mathrm{A}_{2}\left(\mathrm{TXA}_{2}\right)$ by $T$. cruzi . TXA 2 functions as a key regulator of pathogenesis in parasite infection in mice [61]. However, modification of fatty acids by the eicosanoid pathway has not yet been evaluated in the vector stages of $T$. cruzi development.

Usually $10 \%$ of concluded kinomes code for lipidmodulated kinases. These enzymes are continuously regulated by diacylglycerol (DG) and phosphoinositides due to the presence of either a $\mathrm{C} 1$ domain that binds DG, or a pleckstrin homology domain $(\mathrm{PH})$ or $\mathrm{PH}$-like domain that binds 3'-phosphoinositides. Also, in PKC, some C2 domains may bind phospholipids in the presence of calcium [62]. Analysis of the T. cruzi kinome revealed the presence of 190 genes coding for eukaryotic PKs (ePKs). Trypanosomatids have about half of human kinases assigned to the AGC group. This group includes the ePKs that respond to second messengers, including phospholipids and diacylglycerol [63]. However, out of 12 genes only five genes could be assigned to a specific kinase family by sequence, and the remaining seven were not assigned to the PKC family. Due to the ability of the lipid environment to influence $T$. cruzi development, one should look at the structure of lipid kinases in detail. It is likely that some structural differences may be present in the lipid-binding motifs of trypanosomatid lipid kinases, which would provide a promising possibility for the future development of novel chemotherapeutics. Also, different groups have analyzed genes coding for PTPs, which has provided interesting data. The T. cruzi genome codes for only two intracellular, classical PTPs, fully dedicated enzymes for tyrosine dephosphorylation [64-66]. Therefore, if PTPs evolved independently several times, it is likely that protozoan enzymes display unique structural motifs or mechanism of regulation, which could allow for the development of specific chemotherapies [67].

We are living in the T. cruzi post-genomics era where a large amount of genomic information is available, facilitating in silico, proteomics and functional analyses of potential parasite targets for chemotherapy research against Chagas disease. In this context, drugs that act by modulating signal transduction pathways are particularly interesting. Although this matter remains largely unexplored in trypanosomatids, recent work has generated promising data $[60,63,64,68$ 70]. A comparative study of the kinomes of trypanosomatids T. brucei, T. cruzi and Leishmania major showed that approximately $12 \%$ of their kinases are unique to trypanosomatids [63, 69]. Among the protein phosphatases genes, about $40 \%$ are atypical, with no clear orthologs in other eukaryote genomes [64]. The significant differences between T. cruzi and host-cell protein kinases suggest that their specific inhibition may represent a viable therapeutic approach to control Chagas disease. Finally, phosphoproteomic analysis of the epimastigote form of the parasite led to the identification and mapping of 237 phosphopeptides from 119 distinct proteins [70]. The identified phosphoproteins are involved in cell structure, motility, transportation, metabolism, pathogenesis, DNA/RNA/protein turnover and signaling. These phosphoproteomic data provide new insights into the molecular mechanisms governed by protein kinases and phosphatases in $T$. cruzi [70]. The accessible data may now be assayed in terms of parasite biology.
Nevertheless, our understanding of the mechanism of $T$. cruzi growth and differentiation in kissing bugs, with the identification of all of the involved components, their mode of interaction and activated signaling pathways, is far from being complete. In this scenario, multidirectional cellular signaling modulators, either generated from blood digestion or produced by the interaction between the parasite and the vector itself, are strong candidates for the modulation of $T$. cruzi development. This model will provide a huge source of information that certainly will keep us busy for the next 100 years.

\section{ACKNOWLEDGEMENTS}

The authors wish to express their gratitude to Mr. Leonardo José Ribeiro who kindly drew the cartoon depicted in this review. The studies on cell signaling in the authors laboratories were supported by the Brazilian financial agencies Conselho Nacional de Desenvolvimento Cientifico e Tecnologico (CNPq), Fundação de Amparo a Pesquisa Carlos Chagas Filho (FAPERJ) and also by the funding program "Instituto Nacional de Ciência e Tecnologia em Entomologia Molecular".

\section{REFERENCES}

[1] Chagas C. Neue Trypanosomen: Vorla ufige mitteilung. Arch Schiffs-und Trop-Hyg 1909; 13: 120-2.

[2] Chagas C. Nouvelle espe`ce de trypanosomiase humaine. Bull Soc Pathol Exot 1909; 2: 304-7.

[3] Chagas C. New human trypanosomiasis. Morphology and life cycle of Schyzotrypanum cruzi, the cause of a new human disease. Mem Inst Oswaldo Cruz 1909; 1: 159-218.

[4] Urbina JA, Docampo R. Specific chemotherapy of Chagas disease: controversies and advances. Trends Parasitol 2003; 19: 495-501.

[5] Dias JCP, Silveira AC, Schofield CJ. The impact of Chagas disease control in Latin America: a review. Mem Inst Oswaldo Cruz 2002; 97: 603-12.

[6] Wigglesworth VB. The fate of hemoglobin in Rhodnius prolixus (Hemiptera) and other blood-sucking arthropods. Proc R Soci Lond 1943; 131: 313-39

[7] Kappagoda CT, Knapp MF, Linden RJ, Pearson MJ, Whitaker EM. Diuresis from left atrial receptors: effect of plasma on the secretion of the Malpighian tubules of Rhodnius prolixus. J Physiol 1979; 291: 381-91.

[8] Terra WR. Physiology and biochemistry of insect digestion: an evolutionary perspective. Braz J Med Biol Res 1988; 21: 675-734.

Gonzalez MS, Nogueira NF, Mello CB, et al. Influence of brain and azadirachtin on Trypanosoma cruzi development in the vector, Rhodnius prolixus. Exp Parasitol 1999; 92: 100-8.

[10] Nogueira NF, Gonzales M, Garcia EM, de Souza W. Effect of azadirachtin A on the fine structure of the midgut of Rhodnius prolixus. J Invertebr Pathol 1997; 69: 58-63.

[11] Garcia ES, Azambuja P. Development and interactions of Trypanosoma cruzi within the insect vector. Parasitol Today 1991; 7: 240-4.

[12] Kollien AH, Schaub GA. The development of Trypanosoma cruzi in triatominae. Parasitol Today 2000; 16: 381-7.

[13] Azambuja P, Ratcliffe NA, Garcia ES. Towards an understanding of the interactions of Trypanosoma cruzi and Trypanosoma rangeli within the reduviid insect host Rhodnius prolixus. An Acad Bras Cienc 2005; 77: 397-404.

[14] Nogueira NF, Gonzalez MS, Gomes JE, et al. Trypanosoma cruzi: involvement of glycoinositolphospholipids in the attachment to the luminal midgut surface of Rhodnius prolixus. Exp Parasitol 2007; 116: $120-8$.

[15] Boker CA, Schaub GA. Scanning electron microscopic studies of Trypanosoma cruzi in the rectum of its vector Triatoma infestans. Z Parasitenkd 1984; 70: 459-69.

[16] Zeledon R, Bolanos R, Rojas M. Scanning electron microscopy of the final phase of the life cycle of Trypanosoma cruzi in the insect vector. Acta Trop 1984; 41: 39-43. 
[17] Alves CR, Albuquerque-Cunha JM, Mello CB, et al. Trypanosoma cruzi: attachment to perimicrovillar membrane glycoproteins of Rhodnius prolixus. Exp Parasitol 2007; 116: 44-52.

[18] Avila AR, Dallagiovanna B, Yamada-Ogatta SF, et al. Stagespecific gene expression during Trypanosoma cruzi metacyclogenesis. Genet Mol Res 2003; 31: 159-68.

[19] Azambuja P, Guimarães JA, Garcia ES. Hemolytic factor from the stomach of Rhodnius prolixus: evidence and partial characterization. J Insect Physiol 1983; 29: 833-7.

[20] Azambuja P, Mello CB, D'Escoffier LN, Garcia ES. In vitro cytotoxicity of Rhodnius prolixus hemolytic factor and mellitin towards different trypanosomatids. Braz J Med Biol Res 1989; 22: 597-9.

[21] Pereira ME, Andrade AF, Ribeiro JM. Lectins of distinct specificity in Rhodnius prolixus interact selectively with Trypanosoma cruzi. Science 1981; 211: 597-600.

[22] Mello CB, Azambuja P, Garcia ES, Ratcliffe NA. Differential in vitro and in vivo behavior of three strains of Trypanosoma cruzi in the gut and hemolymph of Rhodnius prolixus. Exp Parasitol 1996; 82: 112-21.

[23] Garcia ES, Gonzalez MS, Azambuja P, et al. Induction of Trypanosoma cruzi metacyclogenesis in the hematophagous insect vector by hemoglobin and peptides carrying globin sequences. Exp Parasitol 1995; 81: 255-61.

[24] Naula C, Seebeck T. Cyclic AMP signaling in trypanosomatids. Parasitol Today 2000; 16: 35-8.

[25] Montoreano R, Triana F, Abate T, Rangel-Aldao R. Cyclic AMP in the Malpighian tubule fluid and in the urine of Rhodnius prolixus. Gen Comp Endocrinol 1990; 77: 136-42.

[26] Frainderaich D, Pena C, Isola EL, et al. Stimulation of Trypanosoma cruzi adenyl cyclase by na $\alpha \mathrm{D}$-globon fragment from Triatoma hindgut. Proc Natl Acad Sci USA 1993; 90: 10140-4.

[27] Lehane MJ, Msangi AR. Lectin and peritrophic membrane development in the gut of Glossina m.morsitans and a discussion of their role in protecting the fly against trypanosome infection. Med Vet Entomol 1991; 5: 495-501.

[28] Oliveira PL, Kawooya JK, Ribeiro JM, et al. A heme-binding protein from hemolymph and oocytes of the blood-sucking insect, Rhodnius prolixus. Isolation and characterization. J Biol Chem 1995; 270: 10897-901.

[29] Chou AC, Fitch CD. Hemolysis of mouse erythrocytes by ferriprotoporphyrin IX and chloroquine. Chemotherapeutic implications. J Clin Invest 1980; 66: 856-8.

[30] Schmitt TH, Frezzatti WA, Schreier S. Hemin-induced lipid membrane disorder and increased permeability: a molecular model for the mechanism of cell lysis. Arch Biochem Biophys 1993; 307: 96103.

[31] Gutteridge JM, Smith A. Antioxidant protection by haemopexin of haem-stimulated lipid peroxidation. Biochem J 1988; 256: 861-5.

[32] Paes MC, Oliveira MB, Oliveira PL. Hydrogen peroxide detoxification in the midgut of the blood-sucking insect, Rhodnius prolixus. Arch Insect Biochem Physiol 2001; 48: 63-71.

[33] El-Sayed NM, Myler PJ, Bartholomeu DC, et al. The genome sequence of Trypanosoma cruzi, etiologic agent of Chagas disease. Science 2005; 309: 409-15.

[34] Lara FA, Sant'anna C, Lemos D, et al. Heme requirement and intracellular trafficking in Trypanosoma cruzi epimastigotes. Biochem Biophys Res Commun 2007; 355: 16-22.

[35] Cunha-e-Silva NL, Atella GC, Porto-Carreiro IA, Morgado-Diaz JA, Pereira MG, de Souza W. Isolation and characterization of a reservosome fraction from Trypanosoma cruzi. FEMS Microbiol Lett 2002; 214: 7-12.

[36] Souza CF, Carneiro AB, Silveira AB, et al. Heme-induced Trypanosoma cruzi proliferation is mediated by CaM kinase II. Biochem Biophys Res Commun 2009; 390: 541-6.

[37] Graca-Souza AV, Silva-Neto MA, Oliveira PL. Urate synthesis in the blood-sucking insect Rhodnius prolixus. Stimulation by hemin is mediated by protein kinase C. J Biol Chem 1999; 274: 9673-6.

[38] Alonso A, Sasin J, Bottini N, et al. Protein tyrosine phosphatases in the human genome. Cell 2004; 117: 699-711.

[39] Silveira AB, Castro-Santos J, Senna R, Logullo C, Fialho E, SilvaNeto MA. Tick vitellin is dephosphorylated by a protein tyrosine phosphatase during egg development: effect of dephosphorylation on VT proteolysis. Insect Biochem Mol Biol 2006; 36: 200-9.

[40] Furuya T, Zhong L, Meyer-Fernandes JR, Lu HG, Moreno SN, Docampo R. Ecto-protein tyrosine phosphatase activity in Try- panosoma cruzi infective stages. Mol Biochem Parasitol 1998; 92: $339-48$.

[41] Cosentino-Gomes D, Russo-Abrahão T, Fonseca-de-Souza AL Ferreira CR, Galina A, Meyer-Fernandes JR. Modulation of Trypanosoma rangeli ecto-phosphatase activity by hydrogen peroxide. Free Radic Biol Med 2009; 47: 152-8.

[42] Leite MS, Thomaz R, Oliveira JH, Oliveira PL, Meyer-Fernandes JR. Trypanosoma brucei brucei: effects of ferrous iron and heme on ecto-nucleoside triphosphate diphosphohydrolase activity. Exp Parasitol 2009; 121: 137-43.

[43] Lizenko MV, Regerand TI, Bakhirev AM, Lizenko EI. Comparative biochemical analysis of blood serum lipoproteins from human and various animal species. Zh Evol Biokhim Fiziol 2008; 44: 492500.

[44] Coelho HS, Atella GC, Moreira MF, Gondim KC, Masuda H. Lipophorin density variation during oogenesis on Rhodnius prolixus. Arch Insect Biochem Physiol 1997; 35: 301-13.

[45] Gondim KC, Oliveira PL, Coelho HSL, Masuda H. Lipophorin from Rhodnius prolixus: purification and partial characterization. Insect Biochem 1989; 19: 153-61.

[46] Atella GC, Arruda MA, Masuda H, Gondim KC. Fatty acid incorporation by Rhodnius prolixus midgut. Arch Insect Biochem Physiol 2000; 43: 99-107.

[47] Coppens I, Courtoy PJ. Exogenous and endogenous sources of sterols in the culture-adapted procyclic trypomastigotes of Trypanosoma brucei. Mol Biochem Parasitol 1995; 73: 179-88.

[48] Folly E, Cunha e Silva NL, Lopes AH, Silva-Neto MA, Atella GC. Trypanosoma rangeli uptakes the main lipoprotein from the hemolymph of its invertebrate host. Biochem Biophys Res Commun 2003; 310: 555-61

[49] Atella GC, Bittencourt-Cunha PR, Nunes RD, Shahabuddin M, Silva-Neto MA. The major insect lipoprotein is a lipid source to mosquito stages of malaria parasite. Acta Trop 2009; 109: 159-62.

[50] Cheon HM, Shin SW, Bian G, Park JH, Raikhel AS. Regulation of lipid metabolism genes, lipid carrier protein lipophorin, and its receptor during immune challenge in the mosquito Aedes aegypti. J Biol Chem 2006; 281: 8426-35.

[51] Gomez ML, Erijman L, Arauzo S, Torres HN, Tellez-Inon MT. Protein kinase $\mathrm{C}$ in Trypanosoma cruzi epimastigote forms: partial purification and characterization. Mol Biochem Parasitol 1989; 36: 101-8.

[52] Lammel EM, Barbieri MA, Wilkowsky SE, Bertini F, Isola EL. Trypanosoma cruzi: involvement of intracellular calcium in multiplication and differentiation. Exp Parasitol 1996; 83: 240-9.

[53] Wainszelbaum MJ, Belaunzaran ML, Lammel EM, FlorinChristensen M, Florin-Christensen J, Isola EL. Free fatty acids induce cell differentiation to infective forms in Trypanosoma cruzi. Biochem J 2003; 375: 705-12.

[54] Belaunzaran ML, Lammel EM, Gimenez G, Wainszelbaum MJ, de Isola EL. Involvement of protein kinase C isoenzymes in Trypanosoma cruzi metacyclogenesis induced by oleic acid. Parasitol Res 2009; 105: 47-55.

[55] Sadrzadeh SM, Graf E, Panter SS, Hallaway PE, Eaton JW. Hemoglobin. A biologic fenton reagent. J Biol Chem 1984; 259: 143546.

[56] Graca-Souza AV, Maya-Monteiro C, Paiva-Silva GO, et al. Adaptations against heme toxicity in blood-feeding arthropods. Insect Biochem Mol Biol 2006; 36: 322-35.

[57] Golodne DM, Monteiro RQ, Graca-Souza AV, Silva-Neto MA, Atella GC. Lysophosphatidylcholine acts as an anti-hemostatic molecule in the saliva of the blood-sucking bug Rhodnius prolixus. J Biol Chem 2003; 278: 27766-71.

[58] Mesquita RD, Carneiro AB, Bafica A, et al. Trypanosoma cruzi infection is enhanced by vector saliva through immunosuppressant mechanisms mediated by lysophosphatidylcholine. Infect Immun 2008; 76: 5543-52.

[59] Belaunzaran ML, Wainszelbaum MJ, Lammel EM, et al. Phospholipase A1 from Trypanosoma cruzi infective stages generates lipid messengers that activate host cell protein kinase c. Parasitology 2007; 134: 491-502.

[60] Parodi-Talice A, Duran R, Arrambide N, et al. Proteome analysis of the causative agent of Chagas disease: Trypanosoma cruzi. Int $\mathrm{J}$ Parasitol 2004; 34: 881-6.

[61] Ashton AW, Mukherjee S, Nagajyothi FN, et al. Thromboxane A2 is a key regulator of pathogenesis during Trypanosoma cruzi infection. J Exp Med 2007; 204: 929-40. 
[62] Newton AC. Lipid activation of protein kinases. J Lipid Res 2009; 50 Suppl: S266-S271.

[63] Parsons M, Worthey EA, Ward PN, Mottram JC. Comparative analysis of the kinomes of three pathogenic trypanosomatids: Leishmania major, Trypanosoma brucei and Trypanosoma cruzi. BMC Genomics 2005; 6: 127.

[64] Brenchley R, Tariq H, McElhinney H, et al. The TriTryp Phosphatome: analysis of the protein phosphatase catalytic domains. BMC Genomics 2007; 8: 434

[65] Wilkes JM, Doerig C. The protein-phosphatome of the human malaria parasite Plasmodium falciparum. BMC Genomics 2008; 9: 412 .

[66] Andreeva AV, Kutuzov MA. Protozoan protein tyrosine phosphatases. Int J Parasitol 2008; 38: 1279-95.
[67] Moorhead GB, De Wever V, Templeton G, Kerk D. Evolution of protein phosphatases in plants and animals. Biochem J 2009; 417: 401-9.

[68] Paba J, Santana JM, Teixeira AR, Fontes W, Sousa MV, Ricart CA. Proteomic analysis of the human pathogen Trypanosoma cruzi. Proteomics 2004; 4: 1052-9.

[69] Naula C, Parsons M, Mottram JC. Protein kinases as drug targets in trypanosomes and Leishmania. Biochim Biophys Acta 2005; 1754: 151-9.

[70] Nakayasu ES, Gaynor MR, Sobreira TJ, Ross JA, Almeida IC. Phosphoproteomic analysis of the human pathogen Trypanosoma cruzi at the epimastigote stage. Proteomics 2009; 9: 3489-506.

(c) Silva-Neto et al.; Licensee Bentham Open.

This is an open access article licensed under the terms of the Creative Commons Attribution Non-Commercial License (http://creativecommons.org/licenses/by-nc/3.0/) which permits unrestricted, non-commercial use, distribution and reproduction in any medium, provided the work is properly cited. 